\title{
Non-Curative Resection: Should Clinicians Consider Providing Additional Surgery for All Patients?
}

\author{
Waku Hatta ${ }^{1}$, Takuji Gotoda ${ }^{2}$ and Atsushi Masamune ${ }^{1}$ \\ ${ }^{1}$ Division of Gastroenterology, Tohoku University Graduate School of Medicine, Sendai, ${ }^{2}$ Division of Gastroenterology and Hepatology, \\ Department of Medicine, Nihon University School of Medicine, Tokyo, Japan
}

See "Risk Factors and Clinical Outcomes of Non-Curative Resection in Patients with Early Gastric Cancer Treated with Endoscopic Submucosal Dissection: A Retrospective Multicenter Study in Korea" by Si Hyung Lee, Min Cheol Kim, Seong Woo Jeon, et al., on page 196-205.

According to the Japanese gastric cancer treatment guidelines, when a lesion does not meet the curability criteria for endoscopic resection of early gastric cancers (EGCs), non-curative resection (NCR) is identified, for which additional treatment is recommended in most cases. Thus, it is important to predict NCR and reveal the clinical outcomes of patients with NCR.

In this issue of Clinical Endoscopy, Lee et al. conducted a large-scale retrospective multicenter study in Korea that investigated the risk factors associated with NCR and assessed the long-term clinical outcomes of no additional treatment. ${ }^{1}$ This study demonstrated three clinically important findings: risk factors affecting NCR with endoscopic submucosal dissection, those for local recurrence after NCR, and clinical outcomes of NCR.

Risk factors affecting NCR were old age, undifferentiated histopathology, upper-body tumor location, tumor size $\geq 2 \mathrm{~cm}$, and presence of an ulcer. Tumor size $\geq 2 \mathrm{~cm}$, submucosal invasion, positive horizontal margin, and lymphovascular inva-

Received: February 10, 2020 Revised: February 28, 2020

Accepted: February 28, 2020

Correspondence: Waku Hatta

Division of Gastroenterology, Tohoku University Graduate School of Medicine, 1-1 Seiryo-machi, Aoba-ku, Sendai 980-8574, Japan

Tel: +81-22-717-7171, Fax: +81-22-717-7177, E-mail: waku-style@festa.ocn.ne.jp ORCID: https://orcid.org/0000-0001-9717-0281

cc This is an Open Access article distributed under the terms of the Creative Commons Attribution Non-Commercial License (http://creativecommons.org/ licenses/by-nc/3.0) which permits unrestricted non-commercial use, distribution, and reproduction in any medium, provided the original work is properly cited. sion were risk factors for local recurrence. Although these two findings were already demonstrated in previous studies, ${ }^{2,3}$ it is meaningful that the largest study in Korea confirmed them. It is particularly interesting that lymphovascular invasion is one of the risk factors for local recurrence. Lymphovascular invasion is a known risk factor for lymph node metastasis (LNM) after NCR for EGCs, but it is unclear why lymphovascular invasion is associated with local recurrence. One possible explanation for this is that cancer cells in the lymphatic or venous duct disperse from the duct, leading to local recurrence, but the actual mechanism of local recurrence by lymphovascular invasion has not been clarified. The distinct role of lymphatic and vascular invasion in local recurrence should be revealed.

Lee et al. also revealed the clinical outcomes of NCR. ${ }^{1}$ It is surprising that the overall survival (OS) rate in patients with NCR without additional treatment in this study (98.9\% at 5 years) was much higher than those in previous reports $(72.0 \%-85.0 \%){ }^{4}$ Although the reason for this is unclear, younger age (mean, 62.5 years) might have contributed to the high OS rate in this study.

Although this study contributes to our understanding of NCR, it has a few notable limitations. In this study, the disease-specific survival (DSS) rate of patients with NCR but without additional surgery was significantly lower than that of patients with curative resection, leading to the conclusion that clinicians should consider providing additional treatment after NCR. ${ }^{1}$ However, both DSS rates were high (99.3\% and $99.9 \%$ at 5 years, respectively) and it is unclear whether this 
difference is "clinically" significant. In previous studies, some patients died of gastric cancer despite additional surgery, and in-hospital mortality was observed in $0.36 \%-0.59 \%$ of patients after distal gastrectomy for EGCs according to nationwide data of Japan. ${ }^{6}$ For this reason, DSS rates of patients with and those without additional surgery should be compared to determine the need for additional surgery.

Patients with NCR were not stratified in this study. When piecemeal resection or positive horizontal margin is the only non-curative factor, additional surgery is not the sole treatment strategy because such lesions do not always result in the occurrence of LNM. ${ }^{7}$ For the other category of NCR, further risk stratification by the eCura system was proposed. ${ }^{8}$ This system consisted of five pathological factors with three risk stratifications for predicting LNM and cancer-specific mortality after NCR. ${ }^{8}$ The 5 -year DSS rates of patients with NCR without additional treatment in low-, intermediate-, and highrisk categories in the eCura system were reportedly $99.6 \%$, $96.1 \%$, and $90.1 \%$, respectively. ${ }^{8}$ Therefore, the DSS of each patient category should be further analyzed.

With the continuing aging population, low prevalence of Helicobacter pylori in younger populations, and increasing eradication of $H$. pylori, the number of elderly patients with gastric cancer has increased in Japan and will increase in other Eastern Asian countries including Korea. Thus, the present situation might be a transitional period for gastric cancer to become a disease of elderly patients in these countries. In elderly patients, most mortality cases are non-gastric cancerrelated, ${ }^{9}$ so the current guidelines may not be applicable to all elderly patients. Although it may be appropriate to reconsider the treatment strategy from the balance between the invasiveness of the treatment and the prognosis in elderly patients, few studies are useful for resolving this issue.

This important study clarified various findings of large numbers of patients with NCR. Considering the future perspective of an increasing rate of elderly patients with gastric cancer, the next step may be to investigate the clinical outcomes and establish an algorithm for treating elderly patients with NCR.

Conflicts of Interest

The authors have no financial conflicts of interest.

\section{Author Contributions}

Conceptualization: Waku Hatta

Supervision: Takuji Gotoda, Atsushi Masamune

Writing-original draft: $\mathrm{WH}$

Writing-review\&editing: TG, AM

ORCID

Takuji Gotoda: https://orcid.org/0000-0001-6904-6777

Atsushi Masamune: https://orcid.org/0000-0001-7184-7282

\section{REFERENCES}

1. Lee SH, Kim MC, Jeon SW, Lee KN, Park JJ, Hong SJ. Risk factors and clinical outcomes of non-curative resection in patients with early gastric cancer treated with endoscopic submucosal dissection: a retrospective multicenter study in Korea. Clin Endosc 2020;53:196-205.

2. Eom BW, Kim YI, Kim KH, et al. Survival benefit of additional surgery after noncurative endoscopic resection in patients with early gastric cancer. Gastrointest Endosc 2017;85:155-163.e3.

3. Han JP, Hong SJ, Kim HK, et al. Risk stratification and management of non-curative resection after endoscopic submucosal dissection for early gastric cancer. Surg Endosc 2016;30:184-189.

4. Hatta W, Gotoda T, Koike T, Masamune A. A recent argument for the use of endoscopic submucosal dissection for early gastric cancers. Gut Liver 2019 Sep 30 [Epub]. https://doi.org/10.5009/gnl19194.

5. Hatta W, Gotoda T, Oyama T, et al. Is radical surgery necessary in all patients who do not meet the curative criteria for endoscopic submucosal dissection in early gastric cancer? A multi-center retrospective study in Japan. J Gastroenterol 2017;52:175-184.

6. Yasunaga H, Horiguchi H, Kuwabara K, et al. Outcomes after laparoscopic or open distal gastrectomy for early-stage gastric cancer: a propensity-matched analysis. Ann Surg 2013;257:640-646.

7. Japanese Gastric Cancer Association. Japanese gastric cancer treatment guidelines 2014 (ver. 4). Gastric Cancer 2017;20:1-19.

8. Hatta W, Gotoda T, Oyama T, et al. A scoring system to stratify curability after endoscopic submucosal dissection for early gastric cancer: "eCura system". Am J Gastroenterol 2017;112:874-881.

9. Esaki M, Hatta W, Shimosegawa T, et al. Age affects clinical management after noncurative endoscopic submucosal dissection for early gastric cancer. Dig Dis 2019;37:423-433. 